\title{
Is knowledge and attitude on epidural analgesia during labour satisfactory among pregnant women attending antenatal clinics in Colombo District?
}

\author{
Shehani Lakshika Delwatta ${ }^{1^{*}}$, Pingamage Dona Jayamini Kaushalya ${ }^{1}$, Amugoda Kankanamalage \\ Ravindu Shehan ${ }^{1}$, Chamila Pilimatalawwe ${ }^{2}$, Shamini Prathapan ${ }^{3}$ \\ Medical Student ${ }^{1}$, Senior Lecturer ${ }^{3}$, Department of Community Medicine, Faculty of Medical Sciences, \\ University of Sri Jayawardenapura, Sri Lanka. Consultant Anaesthetist ${ }^{2}$, Sri Jayawardenapura General \\ Hospital, Kotte, Sri Lanka.
}

\begin{abstract}
Labour pain management is a major challenge in maternity care. Epidural analgesia provides safe and effective pain relief during labour. The objective was to assess the knowledge, attitudes and associated factors towards epidural analgesia during labour among pregnant women in Colombo region, Sri Lanka.
\end{abstract}

This was a descriptive study conducted at two selected hospitals in Colombo Sri Lanka. The sample size was 260 and those below $18 \mathrm{yrs}$ were excluded. Participants were provided with a pretested questionnaire. Ethical approval was obtained from Faculty of Medical Sciences, University of Sri Jayewardenepura. Both knowledge and attitude were given separate scores and were categorized as 'good' or 'poor' based on their mean.

Within the study population, $41.9 \%$ had heard of labour analgesia. Among them, only $6.7 \%$ had good knowledge and $15.6 \%$ had a positive attitude towards labour epidural analgesia. The patients with good knowledge about labour epidural analgesia had good attitude towards the procedure $(\mathrm{p}<0.05)$ and there was a positive association between those who had heard of analgesia and their sources of information.

However, as the total level of knowledge and attitudes towards labour epidural analgesia was poor, it is recommended that the health care workers issue pamphlets carrying necessary information following discussions with the pregnant women. After admission they should be given clear information about risks and benefits of the procedure and a post-partum feedback would help in quality improvement.

Keywords: Knowledge; attitude; epidural analgesia; labour pain; maternal care

\section{Introduction}

Even though the intensity of labour pain felt during natural delivery is different from one woman to another, ${ }^{1}$ it is said to be extreme and difficult to cope with where some mothers go on to describe it as the 'worst imaginable pain' they have ever undergone during their lifetime. ${ }^{2}$ This pain can have

*Correspondence: Shehani Lakshika Delwatta

E mail: shehanilakshika@gmail.com

http://www.orcid.org/0000-0002-6059-0706 Received: 28/07/2018

Accepted:24/10/2018

DOI: http://doi.org/10.4038/slja.v27i1.8364

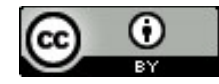

detrimental effects; specially psychological, on both the mother and the fetus. ${ }^{3}$

The American College of Obstetricians and Gynecologists states that anaesthetic services must be available in all hospitals at all times for any mother undergoing labour who desires it. ${ }^{4}$

Due to this, management of labour pain has become one of the main goals of maternity care worldwide ${ }^{5}$ and a considerable amount of research has been done on the subject at international level up to date. Both pharmacological and non-pharmacological methods have been developed to relieve the mothers of labour pain or to help them cope with it better. The non-pharmacological methods including patient education, continuous support during labour, massage therapy, hydrotherapy etc. seem to be 'non-invasive and safer' but there is not 
much research evidence available on their effectiveness. ${ }^{1,6,7}$

The pharmacological methods of labour pain relief include inhalational analgesics such as nitrous oxide and opioids in addition to the regional techniques such as epidural, spinal and combined spinal- epidural block. ${ }^{6,7}$ Of them the epidural is the most commonly used and recommended since it has been shown to be significantly better in pain management than the rest. ${ }^{8}$ Epidural analgesia has also undergone further improvement to reduce the side effects over the recent years with the advancements in the field of medicine. ${ }^{9}$ During epidural analgesia, mothers are not sedated and they will retain both the ability to walk during the first stage of labour and to push effectively during the second. ${ }^{6,7}$ Due to its hypotensive effect it prevents further increase in maternal blood pressure and helps in the management of pre-existing medical conditions such as preeclampsia. ${ }^{7,9}$

While studies have shown that epidural analgesia during labour can give rise to several adverse reactions including increased duration of the second stage of labour, increased need for instrumental delivery, urine retention and maternal pyrexia etc., some of the research findings are ambiguous, mainly because the effects of epidural analgesia cannot be isolated during a delivery. . $^{1,10-13}$

\section{Methodology}

Upon obtaining ethical approval from the Ethics Review Committee of the Faculty of Medical Sciences, University of Sri Jayewardenepura (OTHER 3/17) and Castle Street Hospital for Women (Teaching) (ERC/180/01/2017), Colombo a cross-sectional descriptive study was conducted at the antenatal clinics of Castle Street Hospital for Women and De Soysa Hospital for Women between June-August 2017.

Mothers were requested to complete the selfadministered questionnaire after obtaining their informed consent. Gestational women who were below 18 years and those who refused to participate were excluded. The pre-tested questionnaires initially developed in English, were later translated to Sinhala and Tamil for the easy understanding of the participants.
The questionnaire consisted of three sections. The first section was on socio-demographics (age, residence, religion, education level, occupation, parity, previous count of normal deliveries, pain intensity and the complications during last delivery) while the second and third sections assessed their knowledge level and attitudes towards labour epidural analgesia respectively. On average, each survey took about 10-15 min. The questions were developed following a focused discussion by the authors. A pilot study was done on 10 pregnant mothers prior to conducting the research and no problems were encountered regarding the questionnaire. Each question expressed only one idea (i.e., no questions contained "and") and no questions were phrased in a negative form. Answer types included choosing from a menu of choices or yes/no/don't know. Mothers had the option of writing their own answers to some questions when their answer was not listed.

Total scores were calculated for both knowledge and attitudes sections separately. A score of one was awarded for each correct answer in the knowledge section with a total score of 60 . The mean obtained was 12 and based on this value a score $>=12$ was considered as good knowledge and $<12$ was considered as poor knowledge. The attitude section carried a total score of 80 and was similarly categorized as good and poor attitude based on the mean (48).

At $5 \%$ significance level, assuming $50 \%$ on knowledge and attitudes, the sample size was calculated as 384 . Assuming a $10 \%$ non-response rate, the final sample size was 400 . However, since the actual non-response rate was much higher than anticipated $(34.67 \%)$ the sample size used in the report is 260 . The data obtained were analyzed using SPSS version 23. Associations were tested using Chi square, and the level of significance was set at $<0.05$.

\section{Results}

A total of 260 completed questionnaires were returned with a non-response rate of $34.67 \%$. The mean age of women was 28.12 years and age ranged from 18 to 43 years. All were Sri Lankan and Sinhalese represented the majority (73.1\%). The education level ranged from no formal education to the post graduate level. Women who 
have completed up to advanced level (48.5\%) represented the majority followed by education up to ordinary level (38.8\%). Moreover, the majority of women were unemployed $(70.8 \%)$, and the rest of them were employed either in medical field or in other professions. Furthermore, majority of respondents had a monthly income lower than 50,000 LKR (58.1\%). (Table1)

Table 1: Socio- demographic factors

\begin{tabular}{|c|c|c|c|}
\hline Criteria & Range & $\begin{array}{l}\text { Frequency } \\
(n=260)\end{array}$ & $\begin{array}{l}\text { Percentage } \\
(\%)\end{array}$ \\
\hline \multirow{3}{*}{ Age (years) } & $18-23$ & 53 & 20.4 \\
\hline & $24-29$ & 111 & 42.7 \\
\hline & $>=30$ & 96 & 36.9 \\
\hline \multirow{4}{*}{ Ethnicity } & Sinhala & 190 & 73.1 \\
\hline & Tamil & 34 & 13.1 \\
\hline & Muslim & 35 & 13.5 \\
\hline & Burgher & 1 & .4 \\
\hline \multirow{5}{*}{ Religion } & Buddhist & 161 & 61.9 \\
\hline & Catholic & 38 & 14.6 \\
\hline & Hindu & 24 & 9.2 \\
\hline & Islamic & 35 & 13.5 \\
\hline & Other & 2 & .8 \\
\hline \multirow{4}{*}{ Education } & No formal education & 8 & 3.1 \\
\hline & Up to ordinary level & 101 & 38.8 \\
\hline & Up to advanced levels & 126 & 48.5 \\
\hline & Post graduate & 25 & 9.6 \\
\hline \multirow{3}{*}{ Occupation } & Unemployed/Housewife & 184 & 70.8 \\
\hline & $\begin{array}{l}\text { Employed in the medical } \\
\text { field }\end{array}$ & 3 & 1.2 \\
\hline & Employed in other fields & 73 & 28.1 \\
\hline \multirow{3}{*}{$\begin{array}{l}\text { Monthly } \\
\text { Income }\end{array}$} & $<50,000$ & 151 & 58.1 \\
\hline & $50,000-100,000$ & 63 & 24.2 \\
\hline & $>100,000$ & 46 & 17.7 \\
\hline
\end{tabular}

Of the study population, $58.8 \%$ belonged to the $3 \mathrm{rd}$ trimester while $25 \%$ and $16.2 \%$ belonged to the 2 nd and 3rd trimesters respectively. $58.5 \%$ of the pregnant women were planning to have a normal delivery. When considering the previous delivery count, $62.3 \%$ were primigravida, $23.5 \%$ secundigravida and $14.3 \%$ were multigravida. Of those who had undergone at least one previous normal delivery, $37.1 \%$ had reported a severe pain and $54.6 \%$ a moderate pain during labour but only $36.1 \%$ had been administered labour analgesia and of them only $8.6 \%$ had been given labour epidural analgesia. (Table 2)
Table 2: Pregnancy related factors

\begin{tabular}{|c|c|c|c|}
\hline \multicolumn{2}{|l|}{ Criteria } & $\begin{array}{l}\text { Frequency } \\
(\mathrm{n}=260)\end{array}$ & $\begin{array}{l}\text { Percentage } \\
(\%)\end{array}$ \\
\hline \multirow{3}{*}{ Gestational period } & $1-3$ months & 42 & 16.2 \\
\hline & 4-6 months & 65 & 25.0 \\
\hline & $7-9$ months & 153 & 58.8 \\
\hline \multirow{3}{*}{$\begin{array}{l}\text { Expected type of } \\
\text { delivery }\end{array}$} & Cesarean & 42 & 16.2 \\
\hline & Normal & 152 & 58.5 \\
\hline & Not sure & 66 & 25.4 \\
\hline \multirow{4}{*}{$\begin{array}{l}\text { Previous normal } \\
\text { delivery count }\end{array}$} & None & 162 & 62.3 \\
\hline & One & 61 & 23.5 \\
\hline & Two & 29 & 11.2 \\
\hline & $\begin{array}{l}\text { More than } \\
\text { two }\end{array}$ & 8 & 3.1 \\
\hline \multirow{4}{*}{$\begin{array}{l}\text { *Intensity of labor pain } \\
\text { during previous } \\
\text { delivery }\end{array}$} & No pain & 1 & 1 \\
\hline & Mild pain & 7 & 7.2 \\
\hline & $\begin{array}{l}\text { Moderate } \\
\text { pain }\end{array}$ & 53 & 54.6 \\
\hline & $\begin{array}{l}\text { Severe } \\
\text { pain }\end{array}$ & 36 & 37.1 \\
\hline \multirow{3}{*}{$\begin{array}{l}\text { *Administration of pain } \\
\text { relief during previous } \\
\text { delivery }\end{array}$} & Yes & 35 & 36.1 \\
\hline & No & 44 & 45.4 \\
\hline & Don't know & 18 & 18.6 \\
\hline \multirow{3}{*}{$\begin{array}{l}{ }^{* *} \text { Administration of LEA } \\
\text { during previous } \\
\text { delivery }\end{array}$} & Yes & 6 & 8.6 \\
\hline & No & 18 & 51.4 \\
\hline & Don't know & 14 & 40 \\
\hline
\end{tabular}

Only considering those who had undergone a previous normal delivery.

** Only considering those who had answered 'Yes' for the administration of pain relief during previous delivery.

From the study population, $41.9 \%$ (109 of mothers) had heard about epidural analgesia. 38.8\% (101 of mothers) stated that they were not aware of this procedure. (Table 3)

On further analysis of those who were aware of the procedure $(n=109), 25.7 \%(n=28)$ correctly stated the anatomical location (lower back) for administration of LEA and $17.4 \%$ were aware of administrative personnel of LEA in medical settings. $9.2 \%$ were aware of only one contraindication and $3.7 \%$ knew more than one contraindication regarding LEA. The remaining $87.2 \%$ did not know any contraindication. (Table 3 ) 
Table 3: Knowledge on labour epidural analgesia

\begin{tabular}{|c|c|c|c|c|c|}
\hline \multicolumn{3}{|l|}{ Criteria } & $\begin{array}{l}\text { Freque } \\
\text { ncy } \\
(n=260)\end{array}$ & $\begin{array}{l}\text { Percent } \\
\text { age (\%) }\end{array}$ & $\begin{array}{l}\text { Mean } \\
\text { value }\end{array}$ \\
\hline \multirow{3}{*}{$\begin{array}{l}\text { Awareness } \\
\text { on labor } \\
\text { analgesics }\end{array}$} & \multicolumn{2}{|l|}{ Yes } & 109 & 41.9 & \multirow{3}{*}{ - } \\
\hline & \multicolumn{2}{|l|}{ No } & 101 & 38.8 & \\
\hline & \multicolumn{2}{|c|}{ Not sure } & 50 & 19.2 & \\
\hline \multirow{2}{*}{$\begin{array}{l}{ }^{*} \text { Knowledge } \\
\text { on location } \\
\text { of LEA } \\
\text { administratio } \\
n\end{array}$} & \multicolumn{2}{|c|}{ Knew } & 28 & 25.7 & \\
\hline & \multicolumn{2}{|c|}{ Didn't know } & 81 & 74.3 & - \\
\hline \multirow{2}{*}{$\begin{array}{l}\text { *Awareness } \\
\text { on LEA } \\
\text { administerin } \\
\text { g personnel }\end{array}$} & \multicolumn{2}{|c|}{ Knew } & 19 & 17.4 & \\
\hline & \multicolumn{2}{|c|}{ Didn't know } & 90 & 82.6 & - \\
\hline \multirow{3}{*}{$\begin{array}{l}\text { *Awareness } \\
\text { on } \\
\text { contraindicat } \\
\text { ions for LEA }\end{array}$} & \multirow{2}{*}{$\begin{array}{l}\text { Kne } \\
w\end{array}$} & $\begin{array}{l} \\
\text { answ } \\
\text { er }\end{array}$ & 10 & 9.2 & \multirow{3}{*}{$\begin{array}{l}0.83( \pm \\
2.31)\end{array}$} \\
\hline & & $\begin{array}{l}2 \\
\text { answ } \\
\text { ers or } \\
\text { more }\end{array}$ & 4 & 3.7 & \\
\hline & \multicolumn{2}{|c|}{ Didn't know } & 95 & 87.2 & \\
\hline \multirow{3}{*}{$\begin{array}{l}\text { *Awareness } \\
\text { on benefits } \\
\text { of LEA }\end{array}$} & \multirow[b]{2}{*}{$\begin{array}{l}\text { Kne } \\
w\end{array}$} & $\begin{array}{l}1 \\
\text { answ } \\
\text { ers }\end{array}$ & 26 & 23.9 & \multirow{3}{*}{$\begin{array}{l}1.92( \pm \\
3.1)\end{array}$} \\
\hline & & $\begin{array}{l}2 \\
\text { answ } \\
\text { ers or } \\
\text { more }\end{array}$ & 8 & 7.3 & \\
\hline & \multicolumn{2}{|c|}{ Didn't know } & 75 & 68.8 & \\
\hline \multirow{3}{*}{$\begin{array}{l}\text { *Awareness } \\
\text { on } \\
\text { disadvantage } \\
\text { s of LEA }\end{array}$} & \multirow[b]{2}{*}{$\begin{array}{l}\text { Kne } \\
\text { w }\end{array}$} & $\begin{array}{l}1 \\
\text { answ } \\
\text { ers }\end{array}$ & 15 & 13.8 & \multirow{3}{*}{$\begin{array}{l}1.24( \pm \\
2.73)\end{array}$} \\
\hline & & $\begin{array}{l}2 \\
\text { answ } \\
\text { ers or } \\
\text { more } \\
\end{array}$ & 6 & 5.5 & \\
\hline & \multicolumn{2}{|c|}{ Didn't know } & 88 & 80.7 & \\
\hline \multirow{3}{*}{$\begin{array}{l}\text { *Awareness } \\
\text { on neonatal } \\
\text { benefits of } \\
\text { LEA }\end{array}$} & \multirow[b]{2}{*}{$\begin{array}{l}\text { Kne } \\
w\end{array}$} & $\begin{array}{l}1 \\
\text { answ } \\
\text { ers }\end{array}$ & 23 & 21.1 & \multirow{3}{*}{$\begin{array}{l}1.15( \pm \\
2.22)\end{array}$} \\
\hline & & $\begin{array}{l}2 \\
\text { answ } \\
\text { ers or } \\
\text { more }\end{array}$ & 1 & 0.9 & \\
\hline & \multicolumn{2}{|c|}{ Didn't know } & 85 & 78 & \\
\hline \multirow{6}{*}{$\begin{array}{l}{ }^{*} \text { Total score } \\
\text { on } \\
\text { knowledge }\end{array}$} & \multicolumn{2}{|c|}{ Good } & 10 & 6.7 & \multirow{6}{*}{$\begin{array}{l}\text { 12.12 }( \pm 11 . \\
41)\end{array}$} \\
\hline & \multicolumn{2}{|c|}{ Poor } & 99 & 93.3 & \\
\hline & \multicolumn{2}{|c|}{ Not sure } & 50 & 19.2 & \\
\hline & Didn & now & 81 & 74.3 & \\
\hline & Didn & now & 85 & 78 & \\
\hline & Poor & & 99 & 93.3 & \\
\hline
\end{tabular}

*Considering only those who answered 'Yes' for awareness on labour analgesics. (LEA-Labour Epidural Analgesia)
Considering the contraindications of LEA, $9.2 \%$ of them were only aware of one contraindication, $3.7 \%$ knew more than one and the remaining $87.2 \%$ did not know any contraindications. $23.9 \%$ knew only one benefit, $7.3 \%$ knew more than one benefit and the remaining $68.8 \%$ did not know any benefits regarding LEA. Regarding the disadvantages of LEA, $13.8 \%$ were aware of one disadvantage, $5.5 \%$ knew more than one and $80.7 \%$ did not know any disadvantages. (Table 3)

Among the women who had poor knowledge on epidural analgesia during labour $71 \%$ also had poor attitude and among those who had good knowledge on epidural analgesia during labour, $35 \%$ had poor attitude. This difference was found to be statistically significant $(p<0.05)$. (Table 4$)$

Table 4: Association between knowledge and attitudes towards labour epidural analgesia

\begin{tabular}{|c|c|c|c|c|c|}
\hline & \multicolumn{2}{|c|}{$\begin{array}{l}\text { Knowledge on } \\
\text { Labour Epidural } \\
\text { Analgesia }\end{array}$} & \multirow{2}{*}{$\begin{array}{l}\text { Total } \\
(\%)\end{array}$} & \multirow{2}{*}{$\begin{array}{l}\text { Chi- } \\
\text { Square } \\
\text { value } \\
\text { (df); } p \\
\text { value }\end{array}$} \\
\hline & & $\begin{array}{l}\text { Poor } \\
(\%)\end{array}$ & $\begin{array}{l}\text { Good } \\
(\%)\end{array}$ & & \\
\hline \multirow{2}{*}{$\begin{array}{l}\text { Attitude } \\
\text { on } \\
\text { Labour } \\
\text { Epidural } \\
\text { Analgesia }\end{array}$} & $\begin{array}{l}\text { Poor } \\
(\%)\end{array}$ & $\begin{array}{l}49 \\
(71.01)\end{array}$ & $\begin{array}{l}14 \\
(35.0)\end{array}$ & $\begin{array}{l}63 \\
(57.8)\end{array}$ & \multirow{3}{*}{$\begin{array}{l}13.465 \\
(1) ; \\
0.001\end{array}$} \\
\hline & $\begin{array}{l}\text { Good } \\
(\%)\end{array}$ & $\begin{array}{l}20 \\
(28.9)\end{array}$ & $\begin{array}{l}26 \\
(65.0)\end{array}$ & $\begin{array}{l}46 \\
(42.2)\end{array}$ & \\
\hline \multicolumn{2}{|l|}{ Total (\%) } & $\begin{array}{l}69 \\
(100.0)\end{array}$ & $\begin{array}{l}40 \\
(100.0)\end{array}$ & $\begin{array}{l}109 \\
(100.0)\end{array}$ & \\
\hline
\end{tabular}

Out of the pregnant women who have heard about labour analgesics, $52.3 \%$ had fears in using labour epidural analgesics during their deliveries. Majority of the respondents were confident in their tolerance of pain $(22.9 \%)$ and $16.5 \%$ had fears on the effects of epidural analgesics on the new born. $10.1 \%$ of respondents were afraid of using labour epidural analgesics thinking it would cause future back pain while $9.2 \%$ of study population strongly believed that labour pain is natural and it should not be treated. $7.3 \%$ of study population believed that using labour epidural would cause problems in pushing the baby. $6.4 \%$ believed it would cause future headaches. $5.5 \%$ stated it would have effects on their health. $3.7 \%$ of respondents thought it would cause lower limb weaknesses. (Table 5) 
Table 5: Attitudes towards labour epidural analgesia

\begin{tabular}{|c|c|c|c|}
\hline \multicolumn{2}{|l|}{ Criteria } & \multirow{2}{*}{ 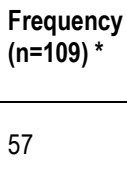 } & \multirow{2}{*}{$\begin{array}{l}\text { Percentag } \\
\text { e (\%) }\end{array}$} \\
\hline \multirow{3}{*}{ Fears regarding LEA } & Yes & & \\
\hline & No & 11 & 10.1 \\
\hline & Not sure & 41 & 37.6 \\
\hline \multirow{3}{*}{$\begin{array}{l}\text { Preference for LEA in } \\
\text { current delivery }\end{array}$} & Yes & 23 & 21.1 \\
\hline & No & 16 & 14.7 \\
\hline & Not sure & 70 & 64.2 \\
\hline \multirow{3}{*}{$\begin{array}{l}\text { Awareness on whether } \\
\text { LEA is offered in their } \\
\text { hospital }\end{array}$} & Yes & 20 & 18.3 \\
\hline & No & 54 & 49.5 \\
\hline & Not sure & 35 & 32.1 \\
\hline \multirow{3}{*}{$\begin{array}{l}\text { If they would specifically } \\
\text { request for LEA if it was } \\
\text { available }\end{array}$} & Yes & 31 & 28.4 \\
\hline & No & 21 & 19.3 \\
\hline & Not sure & 57 & 52.3 \\
\hline \multirow{3}{*}{$\begin{array}{l}\text { If they are willing to bear } \\
\text { the expense of LEA }\end{array}$} & Yes & 26 & 23.9 \\
\hline & No & 24 & 22.0 \\
\hline & Not sure & 59 & 54.1 \\
\hline \multirow{3}{*}{$\begin{array}{l}\text { If they would like to learn } \\
\text { more about LEA }\end{array}$} & Yes & 90 & 82.6 \\
\hline & No & 8 & 7.3 \\
\hline & Not sure & 11 & 10.1 \\
\hline \multirow{3}{*}{$\begin{array}{l}\text { If they would recommend } \\
\text { LEA to family \& friends }\end{array}$} & Yes & 23 & 21.1 \\
\hline & No & 11 & 10.1 \\
\hline & Not sure & 75 & 68.8 \\
\hline \multirow{3}{*}{$\begin{array}{l}\text { If they would encourage } \\
\text { family or friends to learn } \\
\text { about LEA }\end{array}$} & Yes & 62 & 56.9 \\
\hline & No & 8 & 7.3 \\
\hline & Not sure & 39 & 35.8 \\
\hline \multirow{2}{*}{ Total score on attitude } & Good & 46 & 42.2 \\
\hline & Poor & 63 & 57.8 \\
\hline
\end{tabular}

*Considering only those who answered 'Yes' for awareness on labour analgesics.

(LEA-Labour Epidural Analgesia)

$82.6 \%$ of respondents were keen on learning more about epidural analgesics. Even though $56.9 \%$ of pregnant women who have heard about labour analgesics stated that they would encourage others to learn further about LEA, $68.8 \%$ weren't sure whether they would recommend it to their relatives and friends. (Table 5)

There were no statistically significant associations between knowledge or attitude and the criteria that were assessed including age, ethnicity, religion, highest educational qualification, occupation or monthly income. (Table 6)
Table 6: Association between knowledge and attitudes towards Labour Epidural Analgesia and socio- demographic criteria

\begin{tabular}{|c|c|c|c|c|c|c|}
\hline \multirow{2}{*}{ Criteria } & \multicolumn{3}{|c|}{$\begin{array}{l}\text { Knowledge } \\
(n=109)\end{array}$} & \multicolumn{3}{|c|}{$\begin{array}{l}\text { Attitudes } \\
(n=109)\end{array}$} \\
\hline & $\begin{array}{l}\text { Goo } \\
\text { d }\end{array}$ & $\begin{array}{l}\text { Poo } \\
r\end{array}$ & $\begin{array}{l}P \\
\text { value }\end{array}$ & $\begin{array}{l}\text { Goo } \\
\text { d }\end{array}$ & $\begin{array}{l}\text { Poo } \\
r\end{array}$ & $\begin{array}{l}P \\
\text { valu } \\
\mathrm{e}\end{array}$ \\
\hline \multicolumn{7}{|l|}{ Age (years) } \\
\hline $18-23$ & 2 & 15 & \multirow{3}{*}{0.067} & 6 & 11 & \multirow{3}{*}{$\begin{array}{l}0.42 \\
9\end{array}$} \\
\hline $24-29$ & 20 & 29 & & 24 & 25 & \\
\hline$>=30$ & 18 & 25 & & 16 & 27 & \\
\hline \multicolumn{7}{|l|}{ Ethnicity } \\
\hline Sinhalese & 28 & 48 & \multirow{3}{*}{0.801} & 31 & 45 & \multirow{3}{*}{$\begin{array}{l}0.56 \\
2\end{array}$} \\
\hline Tamil & 6 & 13 & & 10 & 9 & \\
\hline Muslim & 6 & 8 & & 5 & 9 & \\
\hline \multicolumn{7}{|l|}{ Religion } \\
\hline Buddhist & 22 & 42 & \multirow{5}{*}{0.631} & 28 & 36 & \multirow{5}{*}{$\begin{array}{l}0.08 \\
9\end{array}$} \\
\hline Catholic & 8 & 8 & & 3 & 13 & \\
\hline Hindu & 4 & 10 & & 9 & 5 & \\
\hline Islamic & 6 & 8 & & 5 & 9 & \\
\hline Other & 0 & 1 & & 1 & 0 & \\
\hline \multicolumn{7}{|l|}{$\begin{array}{l}\text { Highest } \\
\text { educational } \\
\text { qualification }\end{array}$} \\
\hline $\begin{array}{l}\text { No formal } \\
\text { education }\end{array}$ & 1 & 1 & \multirow{4}{*}{0.250} & 0 & 2 & \multirow{4}{*}{$\begin{array}{l}0.50 \\
0\end{array}$} \\
\hline $\begin{array}{l}\text { Up to ordinary } \\
\text { level }\end{array}$ & 11 & 30 & & 16 & 25 & \\
\hline $\begin{array}{l}\text { Up to advanced } \\
\text { levels }\end{array}$ & 25 & 30 & & 26 & 29 & \\
\hline Post graduate & 3 & 8 & & 4 & 7 & \\
\hline \multicolumn{7}{|l|}{ Occupation } \\
\hline $\begin{array}{l}\text { Unemployed/Hous } \\
\text { ewife }\end{array}$ & 21 & 47 & \multirow{3}{*}{0.201} & 24 & 44 & \multirow{3}{*}{$\begin{array}{l}0.15 \\
3\end{array}$} \\
\hline $\begin{array}{l}\text { Employed in the } \\
\text { medical field }\end{array}$ & 2 & 1 & & 2 & 1 & \\
\hline $\begin{array}{l}\text { Employed in other } \\
\text { fields }\end{array}$ & 17 & 21 & & 20 & 18 & \\
\hline \multicolumn{7}{|l|}{ Monthly Income } \\
\hline$<50,000$ & 23 & 41 & \multirow{3}{*}{0.614} & 23 & 41 & \multirow{3}{*}{$\begin{array}{l}0.25 \\
7\end{array}$} \\
\hline $50,000-100,000$ & 11 & 14 & & 12 & 13 & \\
\hline$>100,000$ & 6 & 14 & & 11 & 9 & \\
\hline
\end{tabular}

\section{Discussion}

Majority of the respondents were from the urban areas of Sri Lanka and belonged to the low socioeconomic status. Even though most had secondary level education or beyond, the results of this study showed that only less than half of the pregnant women had previously heard of labour analgesia and of them, less than $20 \%$ were aware about labour epidural analgesia as an option. In comparison to the results obtained in several studies conducted in India and Pakistan which has a similar socio- 
economic status to Sri Lanka, the respondents of the study appeared to be equipped with a similar level of overall knowledge level on labour analgesia. ${ }^{14,15}$, 16

The medical and non-medical sources had contributed to the knowledge level in almost equal percentages in our study and the commonest source of information was a relative or a friend. This

finding resembled the results of other studies conducted in Ireland and India. ${ }^{17}{ }^{18}$ In contrast to the findings from South Africa which reported previous labour experience as a significant contributing factor, awareness was poor within this study population. $^{19}$

Most of the pregnant women had experienced moderate to severe levels of pain during their previous normal deliveries but there was no significant association with the preference for an epidural during the current delivery. Despite their pain perception levels majority of mothers were neither keen on requesting for an epidural analgesic if it wasn't offered by the hospital nor pay for it at their own expense. Confidence in their ability to tolerate pain, harmful effects on the newborn's health and future back pains were among the reasons for rejecting it. A majority had been uncertain about the efficacy of labour epidural analgesia as revealed in a study from Saudi Arabia ${ }^{20}$ but the lack of knowledge was the main reason in others. ${ }^{18,19}$

In the Sri Lankan government hospitals setting pregnant women have the opportunity to request for a labour epidural and even though the mothers can request for an epidural in a non-governmental hospital the request rates are low; probably owing to their low levels of knowledge. More than half of the pregnant women from our study had a poor overall attitude but in comparison; India reported an almost $100 \%$ positive attitude. ${ }^{14}$

It is therefore recommended that the knowledge and attitudes on labour epidurals should be improved especially at the latter stages of the pregnancy, which would lead to patient's decision making for improved delivery and quality of labour care. Further it is recommended that the Ministry of Health and specifically the Family Health Bureau targets a health education session by the Public Health Midwives at the antenatal clinic on the above. A cohort study would be justifiable to observe the pregnant women who had been provided this information and how the attitudes and practices are at the delivery point for women with knowledge on labour epidural analgesics.

\section{References}

1. Jones L. Pain management for women in labour: an overview of systematic reviews. Journal of Evidence-Based Medicine. 2012;5:101-102. https://doi.org/10.1111/j.1756-5391.2012.01182.x PMid:23557475

2. Waldenstrom U, Bergman V, Vasell G. The complexity of labour pain: experience of 278 women. Journal of psychosomatic Obstetrics and Gynecology. 2009. Available from: http://www.tandfonline.com/doi/abs/10.3109/0167 4829609025686 [Accessed 18th May 2017]. (Abstract)

3. Iliadou M. Labour pain and pharmacological pain relief practice points. Health Science Journal. 2009;3(4): 197-201.

4. The American College of Obstetricians and Gynecologists - committee opinion. 687. Approaches to limit intervention during labour and birth. Washington: ACOG; 2017.

5. Simkin P, Bolding A. Updates on Nonpharmacologic approaches to relieve labour pain and prevent suffering. Journal of Midwifery and \& Women's Health.2014;49(6). Available from: http://www.medscape.com/viewarticle/494120 [Accessed 19th May 2017].

https://doi.org/10.1016/j.jmwh.2004.07.007

PMid:15544978

6. Bajwa SK, Bajwa SJS, Singh K, Singh A. Painless labour: how far have we travelled? Sri Lanka Journal of Obstetrics and Gynecology. 2010;32(4):93-98. Available from: http://doi.org/10.4038/sljog.v32i4.3992 [Accessed 19th May 2017]. https://doi.org/10.4038/sljog.v32i4.3992

7. Okojie NQ, Isah EC. Perception of epidural analgesia for labour among pregnant women in a Nigerian tertiary hospital setting. Journal of the West African College of Surgeons. 2014;4(4):142162. Available from:

https://www.ncbi.nlm.nih.gov/pmc/articles/PMC48 66730/ [Accessed 19th May 2017]. PMid:27182515 PMCid:PMC4866730

8. Leighton BL, Halpern SH. The effects of epidural analgesia on labor, maternal and neonatal outcomes: a systematic review. American Family Physician. 2002;186. Available from:

https://www.ncbi.nlm.nih.gov/books/NBK69501/?r eport=classic [Accessed 19th May 2017] (Abstract) 
9. Epidural analgesia in obstetrics - Pros and cons for mother and baby. BMJ. 1989;299:751-752. https://doi.org/10.1136/bmj.299.6702.751

10. Newnham E, McKellar L, Pincombe J. A critical literature review of epidural analgesia. Evidence based Midwifery. 2016:14(1):22-28.

11. Liu EHC, Sia ATH. Rates of caesarean section and instrumental vaginal delivery in nulliparous women after low concentration epidural infusions or opioid analgesia: systematic review. BMJ. 2004;328. Available from:

https://doi.org/10.1136/bmj.38097.590810.7C

[Accessed 20th May 2017]

https://doi.org/10.1136/bmj.38097.590810.7C

12. Weiniger CF, Wand S, Nadjari M, Elchalal U, Mankuta D, Ginosar Y, et al. Post-void residual volume in labor: a prospective study comparing parturients with and without epidural analgesia. Acta Anasthesiologica Scandinavica. 2006;50(10):1297-1303. Available from: https://www.ncbi.nlm.nih.gov/pubmed/16978160[ Accessed 20th May 2017] (Abstract) https://doi.org/10.1111/j.1399-6576.2006.01122.x PMid:16978160

13. Reilly DR, Oppenheimer LW. Fever in term Labour. Journal of Obstetrics and Gynecology Canada. 2005;27(3):218-23. Available from: http://dx.doi.org/10.1016/S1701-2163(16)30513-8 [Accessed on 20th May 2017] https://doi.org/10.1016/S1701-2163(16)30513-8

14. Thakur M, Sagar N, Tandon P. Knowledge and attitude regarding epidural analgesia among expectant mothers. International Journal of Reproduction, Contraception, Obstetrics and Gynecology. 2017 May; 6(5);1929-1931. Available from: http://dx.doi.org/10.18203/2320-

1770.ijrcog20171951 [Accessed on 21st May]. https://doi.org/10.18203/2320-

1770.ijrcog20171951

15. Hazarika R, Rajkhowa T, Parua S. Awareness of epidural labour analgesia among postpartum women at guwahati medical college: a cross sectional study. International Journal of Advanced Health Sciences. 2016;2(10).

16. Minhas MR, Rehana, Afshan G, Raheel $H$. Knowledge, attitude and practice of Parturient regarding Epidural Analgesia for Labour in a university hospital in Karachi. Journal of Pakistan Medical Association. 2005.

PMid:15813631

17. Frohlich S, Tan T, Walsh A, Carey M. Epidural analgesia for labour: Maternal Knowledge, Preferences and Informed Consent. Irish Medical Journal. 2012;104(10):300-2. Available from: http://hdl.handle.net/10147/213509 [Accessed on 21st May 2017].
18. Barakzai A, Haider G, Yousuf F, Haider A, Muhammad N. Awareness of women regarding analgesia during labour. Journal of Ayub Medical College Abbottabad- Pakistan. 2010;22(1):73-75. Available from: http://jamc.ayubmed.edu.pk/index.php/jamc/article /view/3089/1445

19. Mugabe JM, Nel M, Hiemstra LA, Steinberg WJ. Knowledge and attitude towards pain relief during labour of women attending the antenatal clinic of Cecilia Makiwane Hospital, South Africa. South African Family Practice. 2007;49(4):16-16d.

20. Gari A, Aziz A, ALSaleh N, Hamour Y, Abdelal H, Ahmed RS. Awareness of epidural analgesia among pregnant women in Jeddah, Saudi Arabia. Electronic Physician. 2017 May;9(5):4274-80. Available from: http://dx.doi.org/10.19082/4274 [Accessed on 9th October 2017]. https://doi.org/10.19082/4274 